\title{
Análisis de la emisora escolar como propuesta pedagógica y experiencia estudiantil ${ }^{1}$
}

\author{
Lina Andrea Reyes Ramírez ${ }^{2}$
}

Recibido: 14-02-2017

Aceptado: 05-05-2017

\section{RESUMEN}

En el siguiente artículo se presenta un análisis del proyecto de emisora escolar de una institución educativa de la ciudad de Cali, con el fin de dar cuenta por qué se ha mantenido durante diez años, a pesar de no estar constituida como un medio de comunicación profesional, y cuál ha sido su impacto en la comunidad educativa. El análisis de este estudio se realizó bajo las perspectivas teóricas de los usos de la emisora escolar en el aula de clase; la metodología de investigación fue de orden cualitativo; y las categorías de estudio surgieron a partir de las preguntas problema mencionadas anteriormente. Su fundamento principal se centra en que el docente de literatura logra constituir, y posicionar, la emisora escolar como herramienta pedagógica para mejorar competencias argumentativas y expositivas de sus estudiantes mediante la música. Los resultados, en general, muestran que la emisora pasó de tener un carácter informal a concebirse como una herramienta que trasciende del aula de clase, logrando despertar el interés de la comunidad estudiantil.

Palabras clave: emisora escolar, comunidad educativa, proyecto escolar, impacto escolar. el CIER Sur.

2. Comunicadora social - periodista de la Universidad Autónoma de Occidente. Joven investigadora del Grupo de Investigación en Educación de la Universidad Autónoma de Occidente, reconocido por Colciencias.

Correo electrónico: lareyes@uao.edu.co 


\title{
Analysis of the school radio as teaching proposal and student experience
}

\begin{abstract}
The following article analyzes the school radio project of an educational institution in Cali. In order to account why it has remained for ten years despite not being established as a professional communication media and which has been its impact on the educational community. The study analysis was performed under the theoretical perspectives of the use of the school radio in the classroom, the research methodology is qualitative, information sources were interviews, analysis and participant observation, followed by stages of research in which the categories of study that reflect the questions above problems, the principal basis is that literature teacher posicionate the school radio as a teaching tool to improve argumentative and expository skills of their students through music. To finalize the results, analysis of results and the conclusions are given developed.
\end{abstract}

Keywords: School station, educational community, school project, school impact.

\section{Introducción}

Según Marín (2005), la radio es un medio de comunicación que suele ser implementado en las Instituciones Educativas y se conoce bajo el término de "emisora escolar"; es considerado un agente de educación continua por su contribución a la formación integral de los estudiantes en sus ratos de ocio. Entre las virtudes que ofrece la radio, está la formación de opinión, el enriquecimiento de la información y valoración de la misma. En el ámbito escolar, es uno de los medios tecnológicos de mayor acceso, el repertorio didáctico que ofrece es amplio e involucra de manera activa a quienes participan de ella. Sus funciones dentro de una Institución hacen parte de la triada cultura, entretenimiento y educación. La formación de la radio en la escuela, por lo general, se ha orientado 
a enseñar aspectos tales como realización, elaboración de guiones, desarrollo de formatos como el radiodrama y formatos del periodismo. De acuerdo con la autora, en algunas instituciones se toman la labor de dar cuenta de su comunidad e incluso integran a los padres de familia en la construcción de contenidos.

Respecto a las características de la radio, Rodríguez (2001) afirma que es un medio de comunicación ambivalente puesto que es unidireccional y bidireccional en el sentido de que admite la posibilidad de respuesta, instrumental ya que requiere elementos técnicos - a distancia- puesto que se diferencia de la comunicación cara a cara; es colectiva porque tiene múltiples emisores y receptores simultáneos que a diferencia por ejemplo del teléfono, es directa de recepción inmediata en la captación por la audiencia; es reciente, pues utiliza como vehículo físico primario, exclusivamente el sonido; y efímera porque raramente se vuelve al mensaje.

El presente artículo es el resultado de una pregunta de investigación planteada en el marco del proyecto macro "Sistematización de experiencias significativas mediadas por TIC en el CIER Sur: la consolidación de la comunidad docente", desarrollado por la Universidad Autónoma de Occidente y la Universidad del Valle, y tiene como finalidad dar a conocer el por qué la emisora escolar de la Institución Educativa de carácter oficial, Agustín Nieto Caballero, ha logrado mantenerse a lo largo de diez años y cuál ha sido el impacto en su comunidad educativa.

En el año 2012, esta institución escolar fue seleccionada por el Ministerio de Educación Nacional como una de las escuelas innovadoras de Colombia, tras haber participado en una convocatoria a la que enviaron su plan de trabajo y especificaciones de dispositivos tecnológicos. Dicha convocatoria es parte del proyecto Centros de Innovación Educativa Regional CIER, liderado por el Ministerio de Educación Nacional, a través de la Oficina de Innovación Educativa con Uso de Nuevas Tecnologías.

Para desarrollar el tema fue necesario comprender las perspectivas bajolas que las emisoras escolares desarrollan procesos en las instituciones educativas: perspectivas de carácter instrumental, comunicacional o educativo, según el conocimiento, usos y apropiación que se hace de este medio de comunicación (Marín, 2005), esto con el ánimo de identificar el desarrollo que ha atravesado la emisora de la institución educativa en mención y su estado actual. El segundo interrogante, relacionado con el 
impacto en la comunidad educativa, se trabajó a partir de la observación participativa y las entrevistas a los actores de la comunidad estudiantil.

\section{Marco conceptual}

A continuación, se presenta un recorrido teórico que permite comprender, de manera general, a qué hace referencia el término de emisora escolar y qué papel desempeña en las instituciones educativas de acuerdo con la caracterización determinada por sus usos.

De acuerdo con Marín (2005), la emisora escolar o radio escolar es un término adjudicado por las instituciones educativas colombianas que implementan este medio de comunicación. En el marco de las políticas para la radiodifusión en Colombia este término no existe puesto que el Ministerio de Comunicaciones sólo lo reconoce como la aplicación de las ondas electromagnéticas. En Colombia, solo nueve colegios públicos tienen asignadas frecuencias de operación y emisión, que se clasifican en la categoría de "radio de interés público", a diferencia de las radios escolares de Europa y Estados Unidos que disponen frecuencia de bajo alcance en FM para uso escolar.

Un estudio realizado en el año 2003, por la Universidad Nacional de Colombia y la RED del Instituto de Estudios en Comunicación y Cultura -IECO- (citado por Marín, 2005), encontró 223 emisoras escolares entre 419 consultadas. De acuerdo con este estudio, la radio escolar apareció paulatinamente en Bogotá durante las últimas dos décadas, aunque se afirma que la gran mayoría de experiencias son recientes.

Como características generales, la emisora o radio escolar ha sido un tema estudiado por distintos autores, quienes se han enfocado en determinar el impacto que producen estos mensajes emitidos a la sociedad. En primera instancia, la radio escolar es un circuito cerrado de emisión interna a través de parlantes, que en contados casos cuenta con estudios de grabación y algunos en estado de precariedad. Su programación normalmente se estructura a partir de selecciones musicales, actividades de la institución o anuncios de la localidad. La participación en este espacio es de estudiantes y profesores quienes generalmente tienen un interés más personal que institucional (Marín, 2005); en este sentido, los docentes que lideran este tipo de proyectos tienen mayor afinidad con la música y los medios de comunicación. 
Entre otros de los argumentos que destacan el papel de la emisora escolar, varios autores (Jiménez, 2001; Marín, 2005; Carram, 2006; citados por Barrios, 2014), afirman que este es un espacio físico que tienen los jóvenes para comunicarse y a través de este construir una serie de valores en el orden de la democracia, participación, ciudadanía, cultura ciudadana, tolerancia y relaciones interpersonales; a través de la comunicación con los otros. Este conjunto de aspectos enriquecen la formación de los estudiantes pues les permite explorar procesos distintos a los que se viven fuera del aula de clase, se beneficia las relaciones entre ellos y la misma comunidad educativa, resaltando valores y principios que hacen parte de una sana convivencia.

En cuanto a las posibilidades que ofrece la emisora escolar como proyecto de aula, Moreno \& Rosell (2013) afirman que está el presentarse como un proyecto innovador. Múltiples experiencias en España y Latinoamérica han convertido a la radio en un evento cotidiano de la escuela, que a partir del desarrollo de talleres y experiencias de aula brindan soporte a los procesos de aprendizaje o proyectos de educación y comunicación.

Dentro de las ventajas que la emisora escolar ofrece en el aula de clase, Puertas (1989), menciona que es un instrumento de fácil montaje, sencilla manipulación, económica; es un elemento motivador de aprendizaje para los estudiantes, brinda flexibilidad y variedad en los géneros radiofónicos, permite el tratamiento simultáneo del lenguaje oral y escrito, tiene una dimensión comunico - afectiva ya que permite el intercambio de experiencias dentro de la comunidad, posibilita la enseñanza pluridisciplinar y estimula la creatividad.

Pasando al plano de las repercusiones que deja el uso de la emisora escolar, Quintana Garzón (2001) refiere que los estudiantes pueden obtener resultados positivos en distintos planos:

Lingüístico: la práctica radiofónica habitual permite mejorar la expresión oral en sus aspectos formales (entonación, vocalización, comprensión de nuevos términos, mayor sintaxis).

Musical - artístico: se busca la combinación de sonidos más apropiados para cada ocasión, en la que el alumno desarrolla sus propias ideas, gustos y aptitudes. 
Pedagógico: la transmisión de contenido didáctico mediante la manipulación de sonidos refuerza la adquisición de conocimientos y aptitudes.

\section{Personal: la necesidad de trabajar en equipo lleva consigo un alto grado de responsabilidad.}

De acuerdo a estos beneficios, vale la pena resaltar la referencia (Niño y Pérez 2005, citado por Barrios, 2014), en la cual se afirma que la radio les permite a los estudiantes tener una relación lúdica con el conocimiento, que los estudiantes compartan el proceso con sus compañeros, se hablen y escuchen a sí mismos a través de las transmisiones internas o externas del colegio. Este es un ejercicio valioso en el sentido de que se estimula la responsabilidad que tienen los estudiantes con la emisora como en cualquier área académica.

Para concluir, la experiencia de radiodifusión preparada por los alumnos y para los alumnos de una Institución Educativa permite prolongar y enriquecer las enseñanzas de los maestros (Lohr, 1980 citado por Rodríguez 2006). Por tanto, esta herramienta comunicativa es una auxiliar de la educación, siempre y cuando guíe las necesidades de la escuela y sea un medio de expresión de los estudiantes. Es por ello que los docentes son quienes deben usar la radio no solo como una herramienta didáctica que complemente sus clases, sino como un proceso constructivo aplicable.

\section{Perspectivas de uso de la emisora escolar}

A continuación, se plantean las perspectivas de uso de la emisora escolar expuestas por Marín (2005). Las emisoras escolares desarrollan procesos que pueden determinarse en perspectivas de carácter instrumental, comunicacional y en el marco de un proyecto educativo. En el caso de las emisoras de carácter instrumental su objetivo básico es realizar programación musical en el descanso y ofrecer entretenimiento, su selección musical está a cargo del gusto de los estudiantes quienes en ocasiones incluyen comentarios sobre el género o intérprete. También tienen espacio para los mensajes institucionales y los eventos de la institución, su transmisión es a través de altavoces ubicados en el patio del recreo y en pasillos aledaños, estas emisiones suelen ser en directo pues no existe la intención de crear una memoria sonora institucional. 
Este tipo de emisoras cuentan con el apoyo de un docente y en ocasiones del manual de convivencia en donde se encuentra todo lo referente al cuidado de los equipos, normas de comportamiento y uso del lenguaje.

En cuanto a la perspectiva comunicacional, existen emisoras escolares orientadas a promover el desarrollo de las competencias comunicativas y brindar espacios de participación y expresión a los estudiantes. Se caracterizan por el nivel de la elaboración de productos que se reflejan en el resultado de un proceso donde existen tiempos para pensar el tema y llevar un proceso de discusión y modificaciones, dichas prácticas comunicacionales involucran la participación activa de estudiantes y docentes, en algunas instituciones se evidencia que también ha llegado a directivos y demás personal del servicio. Además, la orientación de los docentes garantiza la rotación y capacitación de sus estudiantes en torno a las técnicas y destrezas comunicativas, manejo de software y producción de programas, los cuales giran en torno a la música y los mensajes institucionales, de igual manera brinda el espacio para abordar problemáticas que aquejan a los jóvenes actualmente.

Bajo la perspectiva del marco de un proyecto educativo para vincular el trabajo del aula de clase o con proyectos transversales se busca capacitar a los estudiantes en el uso de la radio, en algunas instituciones se articulan intereses creativos, comunicativos y educativos. Este tipo de emisoras se programan audiciones de música, literatura o mensajes, se hace uso de grabadoras y micrófonos, su programación es segmentada (para niños y para jóvenes), combina lo lúdico y lo pedagógico. Los estudiantes están orientados por los docentes o profesionales en comunicación, existe el proceso de preproducción y producción, apoyan el aprendizaje en las áreas de lenguaje, idiomas y ciencias, también existe una cultura del archivo sonoro pedagógico.

Este tipo de emisoras se orienta a objetivos específicos, entre ellos la prevención de las problemáticas familiares, razón por la que en ocasiones los padres de familia se han visto involucrados en las rutinas de producción y en los ejercicios de audición. La emisora en el marco del proyecto educativo crea espacios de interacción, comunicación, mediación de procesos de aprendizaje, manejo de conflictos, prevención, cualificación personal, familiar y escolar.

En algunos casos surgen alianzas con radios comunitarias o ciudadanas, esta relación ha permitido el surgimiento de colectivos radiofónicos en los 
colegios, lo cual les permite un espacio permanente no solo para divulgar sus actividades sino para desarrollar temáticas infantiles y juveniles. El formato más utilizado por esta radio escolar es la radio - revista, en la que se abordan temas relacionados con medio ambiente y cultura, se trabaja también entrevistas y radiodramas. Hasta el momento, se ha puesto en conocimiento que la emisora escolar presenta distintas posibilidades en el aula de clase, y partiendo de los usos que el docente le otorgue, se puede ubicar bajo dichas perspectivas.

\section{Metodología}

La investigación y su enfoque fueron de orden cualitativo; la primera fuente de información fueron las entrevistas realizadas a los actores principales, bajo un análisis de carácter interpretativo y descriptivo, esto en conjunto con la observación participativa realizada durante el trabajo de campo. En este sentido se estuvo en el contexto de la emisora escolar junto con los actores que participan del proceso, se observó su desarrollo y se interpretaron las percepciones del docente y de sus estudiantes.

\section{Etapas de investigación}

Con el ánimo de resolver los interrogantes mencionados anteriormente, el proceso se dividió en tres segmentos. Para el análisis se presentan, dos categorías de estudio que pretenden dar cuenta de los siguientes interrogantes ¿Por qué la emisora escolar de la institución educativa se ha mantenido a lo largo de diez años? ¿Cuál ha sido su impacto en la comunidad educativa?

La primera categoría se denomina uso que hacen los estudiantes de la emisora escolar, con ello, se pretendió dar a conocer el proceso que desarrollan en torno a este medio e identificar bajo qué perspectiva de uso se encuentra dicho proceso. La segunda categoría se denomina las voces de la emisora, en esta se recopiló el pensar de la comunidad educativa respecto al proyecto de emisora escolar. Partiendo de estos puntos de estudio y de las perspectivas de uso, se dio respuesta a los interrogantes planteados. 


\section{Breve contexto de la emisora escolar}

Para dar un breve contexto, la institución educativa se encuentra ubicada al oriente de Santiago de Cali. Es una institución de carácter oficial, brinda a sus estudiantes educación a través de acciones pedagógicas que fundamentan el ser, el saber y el actuar; posibilitando la interacción armónica con el entorno socio-productivo.

Su emisora nació en el año 2005, con la llegada de tres docentes que vieron en unos bafles la posibilidad de expandir noticias y actividades escolares. En ese entonces su uso se limitaba a las izadas de bandera y reuniones de padres de familia, de ahí surgió la idea de utilizarla más. Eventualmente, se empezaron a hacer programaciones musicales mes a mes, tales como: lunes de música folclórica, martes de música popular, miércoles de música clásica y de ambientación, jueves de combinación entre géneros musicales y viernes dedicado a un cantante específico.

En el año 2009 el docente de Literatura y actual co-investigador del proyecto, recibió la dirección de la emisora escolar y vio en ella la posibilidad de que sus estudiantes mejoraran competencias expositivas y argumentativas, partiendo de la música como tema de interés. Lo anterior, basándose en una experiencia relatada por Fabio Jurado durante un conversatorio, en el que refería, a hacer lo que produce placer y a partir de eso construir saberes. La mayoría de sus alumnos no presentaba los análisis literarios para la clase, entonces el docente decidió llamarlos a preguntar cuáles eran sus gustos, encontró respuestas como Los Aldeanos y Calle 13, decidió proponerles que, en lugar de hacer un análisis a la obra de un autor determinado, lo hiciesen sobre su agrupación musical preferida; el deber del estudiante era leer, investigar y preparar el tema para hacer su exposición a través de la emisora escolar.

La dinámica continuó de la siguiente manera: aquel estudiante al que le gustaba la música de Hector Lavoe, investigaba sobre él y hablaba sobre la temática de la salsa urbana. Durante dos años consecutivos (2013 y 2014), el docente desarrolló el proyecto de emisora en el que consigna como justificación "crear diversos ambientes relacionados con el conocimiento, la investigación, la difusión de valores, el goce artístico y el desarrollo de diversos proyectos pedagógicos. Desde este punto de vista, el proyecto busca convertirse en un importante aliado de todo lo que 
signifique comunicación y desarrollo humano, al mismo tiempo que les entrega a los estudiantes la capacidad de interactuar desde un importante poder mediático, utilizando los recursos y las posibilidades que este puede ofrecer".

También hace mención específica a la música como campo de acción: "se busca la finalidad de difundir diversos géneros musicales, asociados a un enfoque educativo que propende reflexionar sobre géneros musicales, biografías de los artistas y temáticas de las canciones. Este enfoque quiere crear conciencia en la comunidad educativa sobre elementos importantes que la música lleva en sí, como el mensaje de los textos, las características estéticas de los mismos y todo lo relacionado con variedades musicales, tipos de instrumentos y la ejecución de ellos".

En este sentido la emisora escolar empezó a ser reconocida por la institución educativa como una actividad de clase generada por el docente de literatura. Esta iniciativa de vincular los temas de artistas y géneros musicales surgió en el aula de clase a partir de la necesidad mencionada inicialmente, sin embargo, el docente pasó de dictar su curso en el grado once al grado noveno de bachillerato y como esto significaba para los próximos graduandos no participar de dicha actividad, le solicitaron hacer algo al respecto, por ello decidió convertir a la emisora en un proyecto extracurricular que le permitiera a los jóvenes de los distintos grados ser parte de esta.

A lo largo de este breve contexto se ha puesto en evidencia el estado de la emisora escolar en el momento en que el proyecto de "Sistematización de experiencias significativas mediadas por TIC en el CIER Sur: la consolidación docente", inició su curso. Vale la pena resaltar que es una experiencia significativa en el sentido de que han logrado sostener la emisora por tanto tiempo sin tener experiencia o formación alguna en comunicación social. Precisamente por la importancia de profundizar sobre esta situación surgió el interés investigativo de saber por qué la emisora escolar de dicha Institución Educativa se ha mantenido vigente a lo largo del tiempo y cuál ha sido el impacto que ha generado en los jóvenes estudiantes. A continuación, se dará inicio al desarrollo de las respuestas a estos dos interrogantes. 


\section{Resultados}

A continuación se presentan los resultados de la investigación, para dar respuesta a cada cuestionamiento se plantearon dos categorías: uso que hacen los estudiantes de la emisora escolar y las voces de la emisora, las cuales dan cuenta de los interrogantes mencionados en el orden correspondiente.

\section{Primera categoría: uso que hacen los estudiantes de la emisora escolar}

De acuerdo a la reconstrucción que se realizó a partir de cada entrevista que el docente de literatura brindó, se puede afirmar que desde el momento en que él empezó a utilizar la emisora escolar como una herramienta de mediación en el aula de clase, el orden a seguir era que los estudiantes realizaran una investigación previa del género musical o banda elegida, en el caso del rock, buscar sus inicios y realizar una línea de tiempo que registrara su evolución musical. Partiendo de ello, vale la pena resaltar una evolución en cuanto a la preproducción de la audición, puesto que el guión era inexistente. Esta ausencia se trabajó a lo largo del proyecto de investigación macro, Sistematización de experiencias significativas mediadas por TIC: la consolidación de la comunidad docente", puesto que uno de los investigadores, experto en el tema de la radio, dictó talleres en los que se abordó la temática del lenguaje radiofónico y el guión vertical.

La emisora está compuesta por un bafle que se encarga de transmitir a toda la comunidad estudiantil la emisión, una consola de sonido, un computador con acceso a internet y un micrófono de mano. En primera instancia, conectan los parlantes, el micrófono, encienden el ecualizador y dan el saludo de inicio. Las transmisiones que ellos denominan como audición especial, tienen duración de una hora. A continuación, se presenta de manera breve observaciones y descripción de una de sus más recientes audiciones "Que viva la música" de Andrés Caicedo.

Días previos a la actividad, los estudiantes tuvieron una reunión con el docente en la que él les explicó las pautas para elaborar un guion vertical, abordó temáticas tales como el discurso, las dimensiones semánticas y la estética. Esta era la primera ocasión en la que los chicos tendrían este lineamiento para llevar a cabo su audición ya que, como se mencionó 
anteriormente, en otros momentos había sido un poco más espontáneo y no tan estricto como se conoce en el medio radial. La idea era que con base en el libro "Que viva la música" crearan una audición ajustada a dicha estructura.

\begin{tabular}{|c|c|c|c|}
\hline $\mathbf{N}^{\circ}$ & Fuente & Audio & Tiempo \\
\hline 1 & Voz 1 & $\begin{array}{c}\text { Saludo con } \\
\text { introducción }\end{array}$ & 1 minuto \\
\hline 2 & Control & Sonido bestial & 2 minutos \\
\hline
\end{tabular}

Tabla 1. Formato guion vertical.

Por su cuenta los estudiantes siguieron reuniéndose para la elaboración del guión, señalan que fue un trabajo en conjunto en el que discutían sobre qué podían decir acerca de las canciones mencionadas en el libro.

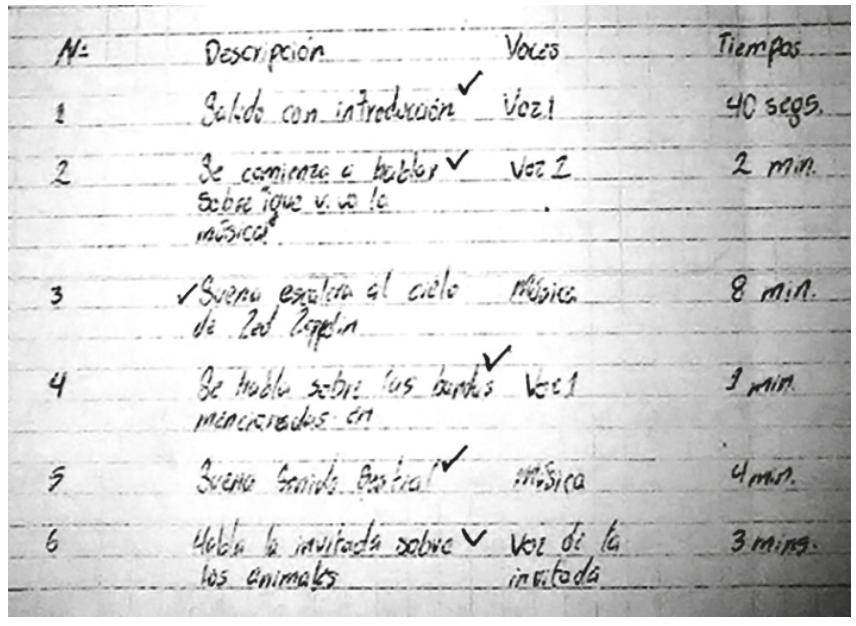

Imagen 1. Segmento de guión vertical, audición "Que viva la música".

Los roles fueron ocupados por los estudiantes de acuerdo a sus intereses:

- Un estudiante fue el encargado de organizar los cables y los equipos.

- Dos estudiantes fueron las voces locutoras.

- Un estudiante el responsable de la música.

- Un estudiante fue el encargado de la seguridad de los equipos.

- Un estudiante fue el encargado de la parte técnica. 
En este sentido, se puede afirmar que el docente no interfirió en las actividades que sus estudiantes decidieron desempeñar. En cuanto al uso de las herramientas de apoyo, YouTube es su reproductor musical permanente, a pesar de ello no se confiaban únicamente de esta plataforma ya que el docente siempre les recomendaba tener una alternativa más, como guardar las canciones a utilizar en un dispositivo móvil.

Las dos voces locutoras dan el saludo de bienvenida, introducen el tema a abordar y entre canciones hablan acerca de la intención que tenía el autor con cada una de ellas y lo que estas significan dentro de la obra de Caicedo Que viva la música. Durante la audición, se tuvo un espacio para abordar el tema del bullyng y una compañera invitada habló acerca de la explotación animal. En términos generales, la actividad giró en torno al guión elaborado, solo en una ocasión saltaron a comerciales, por ende, hubo un pequeño cambio de orden en la estructura de guion planeada inicialmente por los estudiantes.

Partiendo de las observaciones realizadas durante el curso del proyecto de investigación, vale la pena reconocer la evolución que ha tenido la emisora escolar en cuanto a la estructura de su programación, si bien el docente de literatura siempre ha tenido clara la forma en como ha querido direccionar la actividad, la emisora no tenía una construcción radiofónica como tal. La ausencia de guión no les daba un orden temporal de los temas a abordar. Anteriormente los jóvenes se ceñían a lo que encontraban en internet, tenían las páginas separadas y sabían qué les correspondía decir: el cambio se manifestó al aplicar los conocimientos en el uso del guion en el que todo se encuentra consignado de manera ordenada.

En cuanto a la exposición argumentativa y expositiva, competencias que el docente vio fundamental mejorar desde un inicio, evidencia una apropiación de la temática, una investigación rigurosa que les da la herramienta de hablar con conocimiento de causa. En cuanto a la selección de temas para la audición, el docente les da la libertad de escoger lo que quieren abordar en la actividad, en el caso de la audición "Que viva la música" de Andrés Caicedo, fue por interés mutuo pues la lectura había sido trabajada meses atrás en el aula de clase. 


\section{Segunda categoría de análisis: las voces de la emisora}

Las entrevistas realizadas a los distintos actores que giran alrededor de la emisora escolar tuvieron como finalidad comprender sus puntos de vista y percepciones respecto a esta con el ánimo de comprender dicho proceso en la institución educativa.

\section{- Docente de literatura}

Partiendo de la recopilación y la interpretación de las entrevistas hechas al docente, se puede afirmar que esta oportunidad nace a partir de los intereses y gustos musicales de los jóvenes para que se apropien de su discurso, venzan sus temores y se atrevan a hablar en público. De esta manera, se logró llamar la atención de aquellos que por una u otra razón no rendían en sus clases de manera sobresaliente. Uno de los aspectos más productivos que él evidencia es que anteriormente las exposiciones eran muy limitadas y con el uso de la emisora se dio la posibilidad de partir desde el interés del estudiante, que investigue sobre sus gustos y que él como docente pueda encontrar un punto entre lo pedagógico y el proyecto de emisora. El docente de literatura refiere que al estudiante que le gusta el rock le interesaba hablar acerca de Queen y plantear sus argumentos acerca de la banda y lo que dicen en sus letras.

En cuanto a este proceso se resalta el orden y la investigación previa, los estudiantes asumen las funciones y socializan el tema de interés. El hecho de que los alumnos desarrollen mayor sentido de apropiación facilita la intención del docente, que principalmente era la de conseguir que los estudiantes desarrollaran competencias argumentativas y expositivas partiendo de sus gustos musicales para elaborar análisis al mensaje de las letras de las canciones o su propuesta artística. Es esta la razón por la que el docente les sugiere a los jóvenes que partan de un tema de su interés, lo que les interesa del grupo musical y verdaderamente quieren resaltar de este.

\section{- Estudiantes que participan en la emisora escolar}

En el caso de los estudiantes, la visión acerca de la emisora escolar es bastante positiva, en sus testimonios se confirma que es una actividad que realizan con mucho gozo e interés, en la que pueden hablar acerca de sus bandas favoritas. Es evidente el trabajo en equipo que se realiza para la construcción de la audición especial, a pesar de que los roles son 
definidos hay un trabajo mancomunado que resalta cuando están "al aire". La actividad de la emisora escolar también ha permitido que los estudiantes empiecen a considerar la comunicación social como carrera profesional a seguir. Esto ha permitido que cambie la percepción acerca de que no todo es ciencias exactas y que hay distintas posibilidades para explorar y aprender.

En la medida en que los estudiantes aprendieron acerca del lenguaje radiofónico y la manera adecuada en la que se presenta una audición musical, hacen un uso riguroso del guión en el que especifican de manera ordenada los temas a abordar y su acompañamiento musical. En cuanto a la investigación sobre la temática (banda musical o autor), especifican que es ir más allá, comprender qué se quiere manifestar con la letra de la canción, como en las audiciones de The Beatles y "Que viva la música".

Los estudiantes también destacan la creación y el fortalecimiento de los lazos de amistad que se consolidan, gracias al trabajo en equipo y la unión por un mismo propósito. Consideran que el hablar de los temas musicales durante las audiciones especiales es una forma de enviar un mensaje de vida, de aspectos positivos y negativos que permiten hacer reflexionar; de igual manera, lo ven como un ejercicio práctico que les ayuda a vencer el temor de hablar por micrófono.

\section{- Oyentes de la emisora escolar}

En el caso de los estudiantes que participan como oyentes de la emisora escolar, se puede afirmar que disfrutan de dicha actividad en su hora de descanso. En algunos casos hacen alusión a que tengan en cuenta su opinión a la hora de llevar a cabo la programación musical y las audiciones. A pesar de ello, se ha logrado consolidar un espacio en el que cada quien respeta los gustos de sus compañeros. Reiteradamente los jóvenes entrevistados mencionaron que les parecería muy bueno incluir otras temáticas en las audiciones, que no todo girara en torno a la música. La participación de los oyentes se evidencia en que cuando no hay audición especial pueden solicitar sus canciones y los espacios que se les brinda cuando quieren hacer algún anuncio a la comunidad estudiantil.

\section{- Docentes de otras áreas}

En el caso de los docentes que pertenecen a otras áreas, las opiniones están repartidas, algunos lo ven como un espacio que propicia la 
participación estudiantil y otros como un espacio que le quita minutos a sus respectivas clases. Reconocen que esto no es una iniciativa conjunta de un grupo de docentes sino de uno en particular, que tiene afinidad con la música y los medios de comunicación y que ha generado un impacto positivo en los estudiantes.

Algunos, inicialmente veían la emisora como un transmisor musical, ahora reconocen que la dinámica ha cambiado, que hay un proceso detrás de las audiciones especiales. También resaltan el hecho de que los estudiantes tengan su espacio, lo cual se convierte en algo muy importante para ellos.

También hacen referencia al tema del ruido y la falta de control en el volumen lo que en un inicio provocó comentarios negativos ya que entorpecía la armonía de los descansos. Por otra parte, se reconoce que es una mejora a las competencias lingüísticas de los estudiantes y ante las equivocaciones, ellos están ahí para realizar las correcciones pertinentes.

Una de las propuestas que hacen los docentes sobre la emisora versa sobre que se convierta en una dinámica propia de la institución y sus objetivos, que tenga un apoyo desde el concejo directivo, académico y de estudiantes y que también cuente con un fortalecimiento profesional en el área de las comunicaciones para que de esta manera se consolide en el tiempo.

\section{- Directivos}

Las opiniones manifestadas por los directivos tienen como punto en común que saben de una existencia de la emisora, pero no tienen un conocimiento en profundidad sobre ella, saben que los alumnos disfrutan de la música que se programa, que hay una actividad liderada por un docente, a lo cual no se oponen; por el contrario, apoyan ya que es un medio que une a la comunidad estudiantil. Surge la inquietud de que la actividad debería transversalizar otras áreas y no solo literatura, que los docentes aprovechen la herramienta de la emisora para complementar sus clases y divulgar sus actividades, hacer a la comunidad partícipe de lo que se hace en el aula de clase.

Por otra parte, consideran vital la existencia de un documento que sustente cómo se maneja la emisora, qué lineamientos debe seguir para que de esta manera sea un proyecto que se sustente a lo largo de los 
años. Por otro lado, y como sugerencia, creen que es apropiado que desde la emisora se aborden otras temáticas y que no se centre única y exclusivamente en la música. A pesar de que reconocen los obstáculos (infraestructura, conexiones, falta de apoyo de los demás docentes), resaltan que es un proyecto que ha salido adelante gracias a la dedicación del docente de literatura y el buen comportamiento y compromiso de sus estudiantes.

De acuerdo a las categorías de estudio planteadas inicialmente se puede mencionar que en primera instancia, de acuerdo con Marín (2005), y las observaciones e indagaciones hechas durante el proyecto de investigación macro, la emisora escolar de la institución educativa en mención, desde sus inicios se caracterizaba por tener un uso exclusivamente instrumental puesto que servía para transmitir música a gusto de los estudiantes, realizar intervenciones en las izadas de bandera, reunión de padres de familia, día del amor y la amistad, entre otras actividades.

Como la perspectiva lo afirma, en este caso no hay cabida para la intervención de tipo pedagógico, el docente se encarga de que haya orden y los estudiantes de los equipos y de la transmisión musical. Una de las reglas determinadas por el docente en cuanto a programación musical se refiere, es la prohibición de canciones con contenido soez. En cuanto a que esta emisora se guíe bajo los lineamientos del manual de convivencia o algún documento de la Institución no hubo evidencia alguna, sus bases son el respeto y la tolerancia.

Partiendo de estos antecedentes y la ubicación de la emisora escolar bajo una perspectiva instrumental, se evidencia una leve transformación que inicia a partir de la re orientación que le da el docente de literatura con el ánimo de fortalecer competencias en sus estudiantes y buscar que se interesen en la clase al abordar temas de su gusto. Al observar este acompañamiento a los estudiantes, se puede vislumbrar algunos rasgos de una emisora con perspectiva comunicacional, puesto que ellos evalúan los temas de las audiciones, se reúnen, trabajan en equipo y cada quien desempeña un rol, desde la locución hasta el uso de los equipos, sin embargo, hasta ese entonces, el uso del guión estaba ausente.

Durante el marco del proyecto macro "Sistematización de experiencias significativas mediadas por TIC" tuvieron el acompañamiento del investigador, experto en radio escolar y comunitaria lo cual le otorgó 
pautas a los estudiantes sobre el lenguaje radiofónico y guión vertical. Allí hubo una evolución en cuanto a conocimiento se refiere, esto permitió que su trabajo tuviera mayor claridad, orden y se asemejara al proceso rígido de una radio FM ya no había cabida para la improvisación. Muestra de ello se presenta en el segmento "usos de la emisora escolar", en el que se describe el proceso que los estudiantes llevan a cabo a la hora de realizar una audición especial.

En cuanto a las temáticas musicales, el manejo de la información se estructura con momentos claves de la banda musical, sus canciones más importantes o su relación de estas con algún acontecimiento histórico a nivel mundial. También abren espacio para visibilizar temas como el maltrato animal y el bullyng. A lo largo de este proceso, se puede decir que la emisora escolar de dicha institución ha logrado evolucionar en la medida en que se han identificado sus posibilidades. En un inicio el docente logró captar la atención de sus estudiantes partiendo del tema de la música y utilizando la emisora como instrumento de divulgación, al pasar a hacer un uso del guión vertical ya se presenta un proceso que exige preparación, trabajo en equipo y que empieza a vislumbrarse a partir de la perspectiva comunicacional.

Un proceso importante a lo largo de este estudio fue conocer qué piensa la comunidad en general acerca de la emisora escolar. El segmento denominado las voces de la emisora fue el resultado de un análisis a las entrevistas realizadas a lo largo del proyecto macro "Sistematización de experiencias significativas mediadas por TIC", allí se logró evidenciar una similitud entre las perspectivas de los actores, pues si bien es una práctica que valoran y que ha hecho parte de su identidad estudiantil, las opiniones giran en torno a sugerencias como la necesidad de una diversidad de temáticas, es decir, que no se hable únicamente de música, que la emisora sea una dinámica propia de la institución y que se transversalice con distintas áreas; en cuanto a las molestias generadas está en que quita tiempo a las demás clases y que a veces genera mucho ruido.

Por otra parte, se destacan los aspectos positivos que ha brindado a la comunidad educativa, tales como distracción sana para oyentes, aprendizaje para participantes y la inquietud que se genera acerca del futuro profesional. En cuanto al liderazgo de la actividad de emisora escolar, es el docente de literatura quien viene desarrollando este proceso y difiriendo de la perspectiva comunicacional, no se han involucrado directivos ni demás docentes al proyecto escolar. 


\section{Conclusiones}

El desarrollo de la presente investigación surgió a partir del proyecto macro, "Sistematización de experiencias significativas mediadas por TIC en el CIER Sur: la consolidación docente", propuesta generada en el año 2013 por investigadores de la Universidad Autónoma de Occidente y la Universidad del Valle. La experiencia significativa de la institución educativa es el proyecto de la emisora escolar el cual no tenía la estructura que se conoce en el medio profesional. Sin embargo, estaba constituida en la institución años atrás y estaba siendo posicionada como herramienta de un docente en particular. El hecho de que presentara estas características en mención evidenciaba la intención de una estrategia pedagógica que en su momento y sin parámetros ya despertaba el interés de los estudiantes.

La respuesta a las preguntas iniciales planteadas en el transcurso de la investigación, se orientan a concluir que la emisora escolar ha logrado prevalecer a lo largo de los años en la institución gracias a la apropiación de los jóvenes y del docente de literatura. Para ellos, la emisora escolar representa un espacio de convocatoria y aprendizaje en donde se expresan a través de la música y manifiestan temas que competen a sus compañeros en la actualidad.

El punto de partida de la emisora escolar de acuerdo a sus inicios fue instrumental y con el pasar de los años el docente de literatura vio en ella la posibilidad de que sus estudiantes mejoraran competencias argumentativas y expositivas, es por ello que vio en la música el tema ideal para llamar la atención de sus estudiantes. En este sentido, la emisora empieza a evidenciar su propósito dentro de la comunidad educativa, pasa de ser un instrumento para poner música a ser un espacio de jóvenes para jóvenes, en donde cuentan con el apoyo y guía del docente, lo cual fue evidenciado en el segmento descriptivo "uso de la emisora escolar".

De acuerdo a la observación participativa, entrevistas y grabaciones, se evidenció que la emisora escolar se ha mantenido vigente en la institución a pesar de los distintos inconvenientes a lo largo de los años, gracias al compromiso e interés por el aprendizaje que tienen los estudiantes, la voluntad del docente de literatura y la re orientación que le ha otorgado a este espacio, el cual tiene múltiples posibilidades en el aula de clase. 
Su impacto ha sido positivo para la comunidad educativa ya que ha puesto en evidencia el compromiso que tienen los alumnos al invertir tiempo extra y de sus descansos para cumplir con las emisiones en un medio de entretenimiento para la audiencia y un incentivo para las demás generaciones. La convivencia ha sido vital ya que los géneros musicales programados no siempre son del gusto de toda la comunidad educativa, la tolerancia y el respeto han dado el orden para que las transmisiones y audiciones especiales se den sin agresiones e inconvenientes algunos.

Para finalizar, un aspecto a resaltar a lo largo de la investigación que permitió que los estudiantes tuvieran mayor claridad sobre cómo funciona una emisora, fue la asesoría y participación del especialista en temas de la radio, quien se encargó de formar estos aspectos y llenar ciertos vacíos conceptuales. Las audiciones especiales evidenciaron un grado de evolución pues hay una apropiación del tema, se perciben más estructuradas y hay un mayor orden y dominio. Se espera que la actividad crezca y continúe consolidándose bajo una perspectiva comunicacional en la que se haga uso del archivo sonoro y los pregrabados.

\section{Referencias bibliográficas}

Acevedo Zapata, S. (2014). Inclusión digital y educación inclusiva. Aportes para el diseño de proyectos pedagógicos con el uso de tecnologías de la comunicación. Revista De Investigaciones UNAD, 13(1), 41-57. doi:http://dx.doi.org/10.22490/25391887.1130

Amaya Balaguera, Y. (2015). Guía metodológica ágil, para el desarrollo de aplicaciones móviles "AEGIS-MD". Revista De Investigaciones UNAD, 14(1), 97 - 113.

Barrios, G. (junio de 2014). La Radio escolar como experiencia educativa para construir ciudadanía en las escuelas oficiales del Distrito de Cartagena. Castillo, M. 2 o Congreso Internacional de Periodismo Digital SEAP CHILE. Ponencia llevada a cabo en el segundo congreso de periodismo digital, Santiago de Chile, Chile.

Jiménez Mendoza, W., Luciano Alipio, R., \& Soto Carrión, C. (2014). Influencia de las fuentes de financiamiento externo en el crecimiento y desarrollo de las empresas agroindustriales del distrito de Abancay Apurímac Perú (periodo 2012- 2013). Revista De Investigaciones UNAD, 13(1), 261-273. doi:http://dx.doi. org/10.22490/25391887.1141

Jiménez Mendoza, W., Soto Carrión, C., \& Urrutia Huamán, R. (2015). Relación entre modelo PIHEM y gestión educativa en instituciones educativas del nivel inicial en el distrito de Abancay Apurímac Perú, 2014. Revista De Investigaciones UNAD, 14(1), 81 95. doi:http://dx.doi.org/10.22490/25391887.1347 
Marín, O. (2005). La radio. En: Rodríguez. J, Comunicación y escuela: orientaciones para la incorporación, usos y apropiación de los medios de comunicación en las instituciones educativas de Bogotá (pp. 77 - 91) Bogotá: Universidad Nacional de Colombia.

Moreno, I. M., \& Rosell, M. D. M. R. (2013). La radio como recurso didáctico en el aula de infantil y primaria: los podcast y su naturaleza educativa. Tendencias pedagógicas, $21,29-46$.

Puertas. L. (1989). La radio, instrumento motivador para el aprendizaje del Euskera: una experiencia realizada en 3o de EGB. Comunicación, Lenguaje y Educación. 1 (3-4), 53-64.

Quintana, R. (2001). El lenguaje de la radio y sus posibilidades educativas. Comunicar, 9 (17), 97-101.

Régio, L., Egry, E., \& Apostólico, M. (2015). Consideraciones acerca de la importancia del estudio de saberes necesarios para el afrontamiento de la violencia infantil en la atención primaria de salud.. Revista De Investigaciones UNAD, 14(2), 137-149. doi:http://dx.doi.org/10.22490/25391887.1463

Rodríguez, D. (2001). Radio-escuela, una apasionante aventura de comunicación y educación. Comunicar, 9 (17), 97-101.

Rodríguez, H. (2006). Emisoras escolares y ciudadanías. Pereira J.M y Villadiego M, Entre miedos y goces, comunicación, vida pública y ciudadanía. (pp. 274 - 294). Bogotá: Pontifica Universidad Javeriana.

Rojas Mesa, J., \& Leal Urueña, L. (2014). Entre flujos y fronteras: la educación superior mediada tecnológicamente vista a través de una perspectiva etnográfica. Revista De Investigaciones UNAD, 13(2), 9-27. doi:http://dx.doi.org/10.22490/25391887.1143

Silva Carreño, W., \& Beltrán Martin, J. (2015). El rol de género como fundamento humanista de la formación para la ciudadanía. Revista De Investigaciones UNAD, 14(1), 7 - 17. doi:http://dx.doi.org/10.22490/25391887.1343 